\title{
MODEL PREDICTIVE CONTROL OF SYSTEMS WITH COMMUNICATION CONSTRAINTS
}

\author{
Mohamed El Mongi Ben Gaid* Arben Çela* \\ * Design and Control of Industrial Systems Laboratory, \\ ESIEE, Cité Descartes, BP 99, 93162 Noisy-Le-Grand \\ Cedex, France
}

\begin{abstract}
This paper addresses the problem of the on-line scheduling of a limited communication resource in order to optimize the control performance. A multivariable linear system with communication constraints is modeled in the Mixed Logical Dynamical (MLD) framework. The system is controlled using a Model Predictive Controller (MPC), which computes, at each sampling period, the appropriate control values and network allocation. The performance of the controlled system is evaluated using a Linear-Quadratic cost function. At each step, the MPC needs to solve an optimization problem, including logic constraints. The translation of this problem into the Mixed Integer Quadratic Programming (MIQP) formulation is described. Finally, using a numerical example, the relationship between the state variables of the plant and the resultant allocation of the communication resource is investigated. Copyright (C) 2005 IFAC
\end{abstract}

Keywords: Scheduling algorithms, Hybrid systems, Predictive control, Communication networks, Mixed-Integer Programming

\section{INTRODUCTION}

An increasing number of control applications are implemented using distributed architectures, where sensors, controllers and actuators are located in distant nodes and are interconnected through a shared network. In many situations, communication networks are subject to bandwidth limitations. In the automotive industry for example, the Controller Area Network (CAN) bus is widely used because it has many advantages (Lian et al., 2001). A CAN have a maximum bitrate of $1 \mathrm{Mbps}$ (the maximum throughput is less important when the cable length is increased or when a fault-tolerant configuration is used). With this throughput, a distributed control system sampled at $1 \mathrm{~ms}$ and having 16 sensors and 10 actuators exchanging data of size 2 bytes cannot be supported. To solve this problem, engineers may be led to double the sampling period (which may degrade the control performance), to use 2 separate CAN networks for sensors and actuators or to adopt a more expensive technology. But due to the strong competition between the different manufacturers, electronic components cost is a decisive factor in the design process, together with safety, reliability and performance concerns.

Recently, many research works have demonstrated that it is possible to use more efficiently the available computing and network resources if the control design and real-time scheduling design are integrated (Seto et al., 1996; Cervin and Eker, 2000; Branicky et al., 2002; Martí et al., 2002). In (Rehbinder and Sanfridson, 2004), the problem of the stabilization of a linear system subject to communication constraints is considered. In the proposed model, the commands are sent to the actuators through a limited shared TDMA bus. At each slot, only one control command can be 
sent, the remaining commands for the other actuators are held constant. The aims are to optimize off-line both the control and the bus scheduling. The choice of which actuator to update at each slot is handled using the notion of communication sequence (Brockett, 1995). Only periodic communication sequences were considered. Control commands and periodic communication sequences are obtained as a solution of a complex combinatorial optimization problem, which aims at optimizing a linear quadratic cost function. In (Palopoli et al., 2002), the problem of the stabilization of a linear system with distributed groups of actuators is considered. Each group of actuators is connected to the controller through a shared TDMA bus with bandwidth limitations. It is assumed that every TDMA slot, only one command vector can be sent to an actuator group, the other control vectors are set to zero. The stabilization is achieved using a Model Predictive Controller, which allows synthesising online both the appropriate control law and the allocation of the shared bus. The cost function used by the MPC calculates a weighted sum of the infinity norms of the states and the control commands over a specified horizon. The optimization problem solved at each step by the MPC algorithm was proven to be equivalent to the Generalized Linear Complementarity Problem (Ye, 1993).

In this paper, the problem of the Model Predictive Control of a linear system with communication constraints is addressed. The limited communication control problem in formulated the Mixed Logical Dynamical (MLD) theory (Bemporad and Morari, 1999), which provides a framework for modeling and controlling systems where continuous dynamics and logic rules are interdependent. This formulation allows the MPC to calculate both the controls signals and scheduling decisions of the limited communication network. In opposition to (Palopoli et al., 2002), control signals that could not be updated are held constant, a quadratic cost function is used to evaluate the control performance and the ability of the adaptive scheduling to improve the performance of continuous time systems is illustrated.

This paper is organized as follows. In Section 2, the modeling of a multivariable linear system with communication constraints in the MLD framework is describted. A Model Predictive Controller for this limited communication control system is designed in Section 3. This controller needs to solve a constrained optimization problem at each step. Section 4 shows how such a problem can be translated into the MIQP formulation. Finally, in Section 5, a numerical example is used to illustrate the effectiveness of the approach and to study the relationship between control and scheduling aspects in the proposed model.

\section{PROBLEM FORMULATION}

Consider the continuous-time LTI system described by the state space equation:

$$
\dot{x}(t)=A_{c} x(t)+B_{c} u(t)
$$

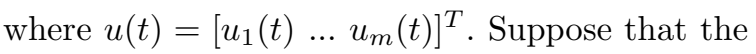
system is controllable and that the entire state vector is measurable. One can assume, without loss of generality, that the system contains $m$ distributed actuators, and that the control signal $u_{k}$ is transmitted to the $k^{t h}$ actuator. A discretetime controller is used in control. The control commands are sent to the actuators through a shared communication resource, which is constrained to have a limited bandwidth. The communication resource is limited in the sense that, at each sampling interval, it can carry at most $b$ control commands, where $b \leq m$ (Hristu, 1999). The adopted modeling allows to specify separately the temporal parameters that are related to the dynamics of the control system (the sampling period) and those corresponding to the network capacity (the network bandwidth), and thus permits to achieve a maximal use of the available network resources.

In order to derive a control law for the system, a discrete-time representation of (1) is used :

$$
x(k+1)=A x(k)+B u(k)
$$

The constraints affecting the transmission of control commands to actuators can be modeled by introducing a vector $\delta$ of $m$ binary variables $\delta_{1}, \ldots, \delta_{m}$ such that:

$$
\delta_{i}(k)=1 \Longleftrightarrow u_{i}(k) \text { is updated at instant } k
$$

The bandwidth limitation can be described by the following inequality :

$$
\sum_{i=1}^{m} \delta_{i}(k) \leq b
$$

The control commands that cannot be updated at the $k^{\text {th }}$ sampling period are held constant. This assertion can be modeled using the logic formula:

$$
\delta_{i}(k)=0 \Longrightarrow u_{i}(k)=u_{i}(k-1)
$$

Finally, suppose that control signals are limited:

$$
l_{i} \leq u_{i} \leq U_{i}
$$

and that $l_{i}<0$ and $U_{i}>0$.

The proposed model of the control system with limited communication, includes a recurrent equation (2) describing the dynamics of the system, saturation constraints affecting control signals (6), inequality constraints (4) modeling the limitations of the communication medium and logic rules (5) addressing the interdependence between control signals $\left(u_{i}\right)$ and scheduling decisions $\left(\delta_{i}\right)$. This model can be handled using the Mixed Logical Dynamical theory (MLD) (Bemporad and Morari, 1999). 


\section{MODEL PREDICTIVE CONTROLLER DESIGN}

Different feedback-control strategies were proposed in order to control MLD systems, among these strategies, Model Predictive Control (MPC) was choosen. This choice was motivated by:

- The need to optimize simultaneously control actions and network scheduling, in order to achieve a better quality of control than the static network allocation schemes.

- The need for a control law that changes online the sampling period in order to improve the quality of control. This requires that these variations are taken into account by the control law (Martí et al., 2001).

Using Model Predictive Control, an optimal control problem is solved on-line at each sampling period. It aims at finding the optimal control values sequence $v^{N-1}=(v(0), \ldots, v(N-1))$ and the optimal network allocation sequence $\sigma^{N-1}=$ $(\sigma(0), \ldots, \sigma(N-1))$ which are solutions of the following optimization problem:

$\left\{\begin{array}{l}\min _{v^{N-1}, \sigma^{N-1}} \sum_{h=0}^{N} y^{T}(h) Q y(h)+\sum_{h=0}^{N-1} v^{T}(h) R v(h) \\ \text { subject to: } \\ y(0)=x(k) \\ y(h+1)=A y(h)+B v(h), h \in[0, N-1] \\ \sum_{i=1}^{m} \sigma_{i}(h) \leq b, h \in[0, N-1] \\ \sigma_{i}(0)=0 \Longrightarrow v_{i}(0)=u_{i}(k-1) \\ \sigma_{i}(h)=0 \Longrightarrow v_{i}(h)=v_{i}(h-1), h \in[1, N-1]\end{array}\right.$

where $Q, R>0$. The solution of this problem is based on the prediction of the future evolution of the system over an horizon of $N$ sampling periods. This predicted evolution is calculated according to the model of the plant, knowing the current state $x(k)$ of the system. The variables $y(h), h \in[0, N]$ represent the predicted values of system states $x(k+h)$. The sequences $(v(0), \ldots, v(N-1))$ (virtual control sequence) and $(\sigma(0), \ldots, \sigma(N-1))$ (virtual network allocation sequence) are called virtual sequences, because they are based on the predicted evolution of the system. The resolution of this problem aims at finding the optimal virtual control sequence $\left(v^{*}(0), \ldots, v^{*}(N-1)\right)$ and the optimal virtual network allocation $\left(\sigma^{*}(0), \ldots, \sigma^{*}(N-1)\right)$ which minimize a quadratic cost function over a finite horizon of $N$ sampling periods. Assuming that the optimal virtual sequences exist, the actual control commands are obtained by setting:

$$
u(k)=v^{*}(0)
$$

and:

$$
\delta(k)=\sigma^{*}(0)
$$

and disregarding the remaining elements of the sequences $\left(v^{*}(1), \ldots, v^{*}(N-1)\right)$ and $\left(\sigma^{*}(1), \ldots, \sigma^{*}(N-\right.$ $1)$ ). Only the control commands $u_{i}(k)$ verifying $\delta_{i}(k)=1$ are sent to the actuators. At the next sampling period (step $k+1$ ), the whole optimization procedure is repeated, based on $x(k+1)$.

A important issue concerns the stability of the proposed Model Predictive Controller. If the following constraint is added to problem (7):

$$
y(N)=0
$$

the following result is obtained :

Theorem 1. If at $k=0$, a feasible solution exists for the problem (7) augmented with the additional constraint (10). Then $\forall Q=Q^{T}>$ $0, R=R^{T}>0$, the MPC law $(7)(10)(8)(9)$ stabilizes the system (2) such that:

$\lim _{k \rightarrow \infty} x(k)=x_{e}=0$

$\lim _{k \rightarrow \infty} u(k)=u_{e}=0$

Proof: For $\delta=\delta_{e}=0$ and $u=u_{e}=0$, the constraints (4), (5) and (6) are verified. It follows that $\delta$ is definitely admissible. The remaining of the proof is obtained by the direct application of the sufficient stability conditions for the Model Predictive Control of MLD systems stated in (Bemporad and Morari, 1999).

\section{FORMULATION OF OPTIMIZATION PROBLEM IN THE MIQP FORM}

In addition to linear inequalities, problem (7) involves logical constraints. In order to solve this optimization problem, these logical formulas need to be translated into linear equalities and inequalities. Remarking that:

$$
\sigma_{i}(h)=0 \Longrightarrow v_{i}(h)=v_{i}(h-1)
$$

is equivalent to:

$$
v_{i}(h)-v_{i}(h-1)=\sigma_{i}(h) v_{i}(h)-\sigma_{i}(h) v_{i}(h-1)
$$

and using the procedure described in (Bemporad and Morari, 1999), the product of continuous and logical variables can be translated into linear inequalities, which however requires the introduction of auxiliary variables. For example, if $l_{i}$ and $U_{i}$ are respectively the lower and the upper bounds of $v_{i}(h)$, the expression $z_{i}^{v}(h)=\sigma_{i}(h) v_{i}(h)$ is equivalent to:

$$
\begin{aligned}
& z_{i}^{v}(h) \leq U_{i} \sigma_{i}(h) \\
& z_{i}^{v}(h) \geq l_{i} \sigma_{i}(h) \\
& z_{i}^{v}(h) \leq v_{i}(h)-l_{i}\left(1-\sigma_{i}(h)\right) \\
& z_{i}^{v}(h) \geq v_{i}(h)-U_{i}\left(1-\sigma_{i}(h)\right)
\end{aligned}
$$

Note that if $\xi_{i}(h)=v_{i}(h-1)$, the same procedure can be applied to $z_{i}^{\xi}(h)=\sigma_{i}(h) \xi_{i}(h)$. 
Observing that $\forall h \in[0, N], y(h)$ is only a function of $x(k)$, the expression of cost function can be simplified. Let:

$$
V=\left[\begin{array}{c}
v(0) \\
\vdots \\
v(N-1)
\end{array}\right]
$$

the cost function can be written:

$$
J=V^{T} H V+2 f^{T} V+c
$$

where $H=\left[H_{i, j}\right]_{0 \leq i \leq N, 0 \leq j \leq N}$, is a symmetric $m . N \times m . N$ matrix, containing $N^{2}$ sub-matrices $H_{i, j}$ of size $m \times m$ such that:

$$
H_{i, j}=\delta_{i j} R+\sum_{l=0}^{N-1-\max (i, j)} B^{T}\left(A^{T}\right)^{l} Q A^{|i-j|+l} B
$$

$\delta_{i j}$ stands for the Kronecker symbol. $f=\left[f_{1} \ldots f_{N}\right]^{T}$ is an $N . m$ vector containing $N$ vectors $f_{i}$ of size $m$ such that:

$$
f_{i}=\sum_{l=i}^{N} x^{T}(k)\left(A^{T}\right)^{l} Q A^{l-i} B
$$

and c is a constant: $c=\sum_{l=0}^{N} x^{T}(k)\left(A^{T}\right)^{l} Q A^{l} x(k)$.

Let: $\Xi=\left[\begin{array}{c}\xi(0) \\ \vdots \\ \xi(N-1)\end{array}\right], \Sigma=\left[\begin{array}{c}\sigma(0) \\ \vdots \\ \sigma(N-1)\end{array}\right]$, $Z^{v}=\left[\begin{array}{c}z^{v}(0) \\ \vdots \\ z^{v}(N-1)\end{array}\right], Z^{\xi}=\left[\begin{array}{c}z^{\xi}(0) \\ \vdots \\ z^{\xi}(N-1)\end{array}\right]$ and $\mathcal{V}=\left[\begin{array}{c}V \\ \Xi \\ \Sigma \\ Z^{v} \\ Z^{\xi}\end{array}\right]$ the problem $(7)$ can be rewritten in the form:

$$
\left\{\begin{array}{l}
\min _{\mathcal{V}} \frac{1}{2} \mathcal{V}^{T} G \mathcal{V}+g^{T} \mathcal{V} \\
\mathcal{A} \mathcal{V} \leq \mathcal{B}
\end{array}\right.
$$

where $G, g, \mathcal{A}$, and $\mathcal{B}$ can be easily found. Problem (18) is called Mixed Integer Quadratic Program (Lazimy, 1985). Many academic and commercial solvers are available for this particular problem.

\section{A NUMERICAL EXAMPLE}

In order to illustrate the proposed approach and to study the interdependency of control and scheduling, especially the relationship between the state space vector of the plant and the network allocation, consider the continuous-time LTI system described by the state matrix $A_{c}=\left[\begin{array}{cc}A_{c_{1}} & 0 \\ 0 & A_{c_{2}}\end{array}\right]$ and the input matrix $B=\left[\begin{array}{cc}B_{c_{1}} & 0 \\ 0 & B_{c_{2}}\end{array}\right]$, where $A_{c_{1}}=A_{c_{2}}=\left[\begin{array}{cc}3 & -1 \\ 2 & 0\end{array}\right]$ and $B_{c_{1}}=B_{c_{2}}=\left[\begin{array}{l}1 \\ 0\end{array}\right]$. The system consists of two identical and independent subsystems, which are open-loop unstable. A scalar control input is used in order to stabilize each subsystem.

The system is stabilized using a Model Predictive Controller. The MPC law is based on a discrete model obtained at the sampling period of $T_{s}=$ $0.1 s$. The weighting matrices are chosen such as $Q=100 \operatorname{diag}(1,1,1,1)$ and $R=\operatorname{diag}(1,1)$. The MPC horizon is set to $N=15$. The two control inputs $u_{1}$ (corresponding to the first subsystem) and $u_{2}$ (corresponding to the second subsystem) are sent to the corresponding actuators through a shared communication network. The network bandwidth is such that only a control input can be transmitted each sampling period, thus $b=1$. In this particular simple example, a network slot time is equal to the sampling period of the system. $u_{1}$ and $u_{2}$ are subject to saturation constraints: $-10 \leq u_{1} \leq 10$ and $-10 \leq u_{2} \leq 10$.

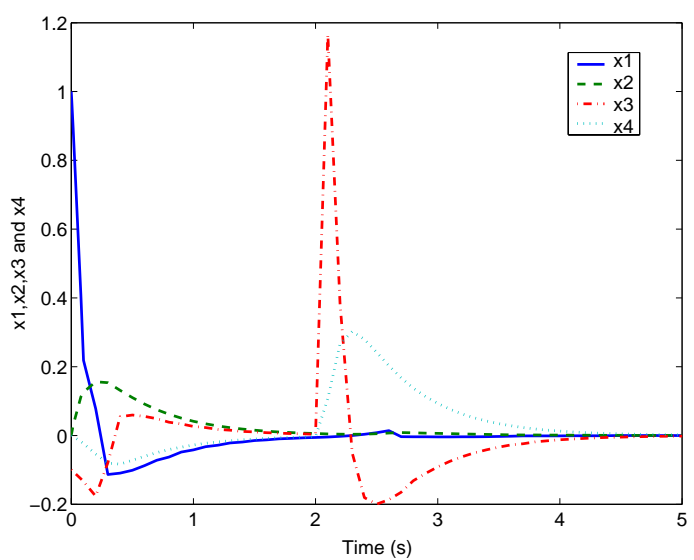

Fig. 1. Evolution of the state variables of the two subsystems

Figure 1 illustrates the evolution of the state variables of the first subsystem $\left(x_{1}\right.$ and $\left.x_{2}\right)$ and those of the second subsystem $\left(x_{3}\right.$ and $\left.x_{4}\right)$. The initial

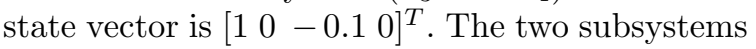
converge progressively to the equilibrium state. At $t=2 s$, the second subsystem is disturbed (the state variables $x_{3}$ and $x_{4}$ pass suddenly to respectively 1.18 and 0.28 ). These deviations are corrected and the second subsystem is stabilized at $t=5 \mathrm{~s}$.

To understand the interaction between control and scheduling aspects, consider the network schedule depicted in figure 2. The lower signal corresponds to $\delta_{1}$ and the upper signal to $\delta_{2}$ $\left(\delta_{i}=1\right.$ means that the network is dedicated to the transmission of control signal $u_{i}$ ). The network schedule shows how the MPC algorithm allocates the communication resources to each subsystem. 


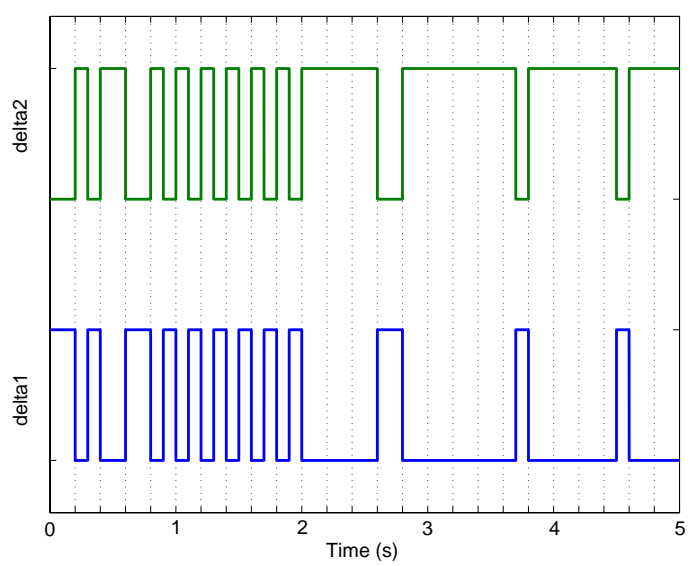

Fig. 2. Network schedule

At $t=0 \mathrm{~s}$, the first subsystem has the greatest deviation from equilibrium position. In order to optimize the cost function, the MPC law allocates the two first slots to the transmission of the control signal $u_{1}$. Next, when the two subsystems reach approximately the same "distance" from the equilibrium, the network bandwidth is allocated fairly. At $t=2 s$, the second subsystem is disturbed, the MPC control law reacts and allocates six consecutive slot times to the transmission of the control signal $u_{2}$. Because the first subsystem is closer to the equilibrium than the second subsystem, the remaining slots are mainly dedicated to the transmission of $u_{2}$.

In order to evaluate the effectiveness of this approach, the proposed adaptive scheduling scheme is compared to the static fair allocation of the network, where the control values $u_{1}$ and $u_{2}$ are sent alternately on the network. To devise the control law for the system which is scheduled according to the static fair network allocation, the continuous system has to be sampled with a sampling period of a least 0.2 seconds. The Model Predictive Controller uses the discrete model obtained at this sampling frequency. The prediction horizon is the same for the two schemes.

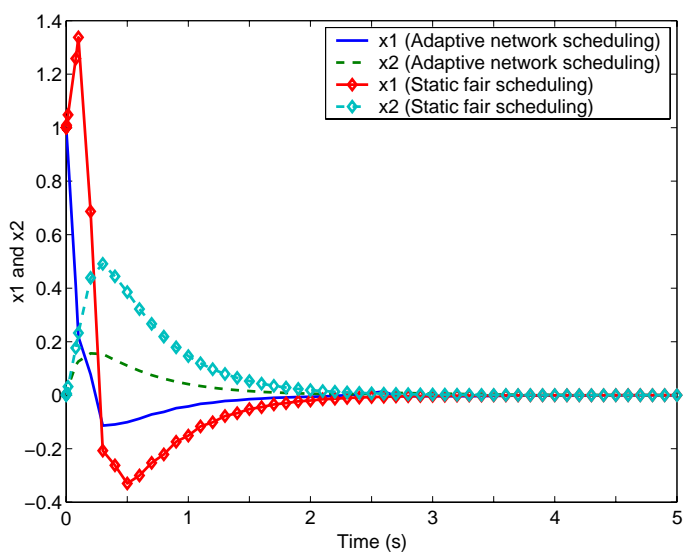

Fig. 3. Comparaison between the control performance results of the adaptive and static network scheduling schemes, states $x_{1}$ and $x_{2}$
The two systems are simulated starting from the same initial conditions as above. At $t=2 s$, they are subjected to the same disturbance. The simulation results related to $x_{1}$ and $x_{2}$ are depicted in figure 3 and those related to the states $x_{3}$ and $x_{4}$ in figure 4 . These results show that significant improvements of the control performance are achieved by the adaptive scheduling scheme, compared to the static fair network allocation. These improvements are due to the ability of the adaptive scheduling scheme to react earlier and quicker to the disturbances. In figure 3 , the adaptive scheduling algorithm improves the global quality of control, by allocating the two first slots to subsystem 1 , which have the greatest deviation from the equilibrium position, in opposition to the static fair scheduling algorithm, which allocates the first slot independently of the dynamical state of the subsystems. In figure 4 and at $t=2 s$, instead of reserving half of the network bandwidth to subsystem 1, which is very close to the equilibrium, the adaptive scheduling algorithm uses these resources to achieve a quicker and better stabilization of subsystem 2. These results are is accordance with those of (Martí et al., 2002), where the empirical study of the relationship between resource allocation and control performance was undertaken.

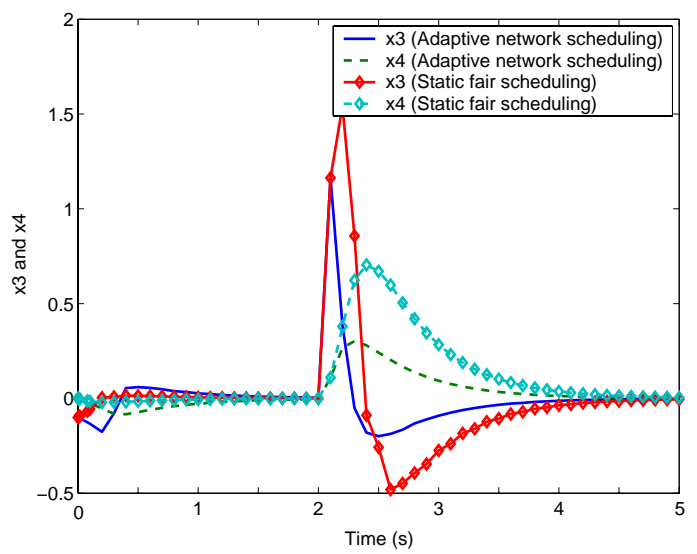

Fig. 4. Comparaison between the control performance results of the adaptive and static network scheduling schemes, states $x_{3}$ and $x_{4}$

\section{IMPLEMENTATION ASPECTS}

MPC techniques were mainly applied to systems with slow dynamics, because they require a very important computational effort due to the online solving of the involved optimization problem. However, many advances have been made since the development of the first MPCs such as explicit MPC, which allows to move off-line the most important part of the computational complexity of the optimization algorithm, enabling the application of MPC techniques to fast systems, like those 
addressed in this paper. The idea of explicit MPC for hybrid systems comes from the observation that the solution to the finite time optimal control problem (based on linear or quadratic cost functions) is a time varying piecewise affine state feedback control law (Bemporad et al., 2002). If the state $x(k)$ is considered in the optimization problem as a parameter, the use of multiparametric optimization techniques (Bemporad et al., 2000) allows the solving of the optimization problem for all the feasible values of $x(k)$. The building of the explicit solution is essentially based on the partitioning of the state space. The on-line computation of the control low is reduced to finding the partition which contains the current state vector and then to calculate an affine function of this state. We are currently investigating the use of multiparametric optimization techniques to find sub-optimal solutions to our problem formulation.

\section{CONCLUSIONS}

This paper addressed theoretically the problem of the on-line scheduling of control applications with communication constraints. An approach where the scheduling is based on the information related to the state space variables of the system was proposed. In this approach, control and scheduling are tightly coupled in order to achieve a better control performance thanks to a more efficient use of the available network resources. In opposition to other approaches where the co-design of control and scheduling aims at optimizing steady state performance, transient behavior is the main concern of the proposed approach.

\section{REFERENCES}

Bemporad, A. and M. Morari (1999). Control of systems integrating logic, dynamics, and constraints. Automatica 35(3), 407-427.

Bemporad, A., F. Borelli and M. Morari (2000). Piecewise linear optimal controllers for hybrid systems. In: Proceedings of the 19th American Control Conference. Chicago, USA.

Bemporad, A., F. Borelli and M. Morari (2002). On the optimal control law for linear discrete time hybrid systems. In: Proceedings of the 5th International Workshop on Hybrid Systems: Computation and Control. Stanford, California, USA.

Branicky, M.S., S.M. Phillips and W. Zhang (2002). Scheduling and feedback co-design for networked control systems. In: Proceedings of the 41st IEEE Conference on Decision and Control. Las Vegas, USA.

Brockett, R. (1995). Stabilization of motor networks. In: Proceedings of the 34th IEEE Con- ference on Decision and Control. New Orleans, Los Angeles, USA.

Cervin, A. and J. Eker (2000). Feedback scheduling of control tasks. In: Proceedings of the 39th IEEE Conference on Decision and Control. Sydney, Australia.

Hristu, D. (1999). optimal control with limited communication. PhD thesis. Division of Engineering and Applied Sciences, Harvard University.

Lazimy, R. (1985). Improved algorithm for mixedinteger quadratic programs and a computational study. Mathematical Programming 12, 100-113.

Lian, F.-L., J.R. Moyne and D.M. Tilbury (2001). Performance evaluation of control networks: Ethernet, controlnet, and devicenet. IEEE Control Systems Magazine 21(1), 66-83.

Martí, P., J.M. Fuertes, G. Fohler and K. Ramamritham (2002). Improving quality-of-control using flexible timing constraint : Metric and scheduling issues. In: Proceedings of the 23rd IEEE Real-Time Systems Symposium. Austin, Texas, USA.

Martí, P., J.M. Fuertes, K. Ramamritham and G. Fohler (2001). Jitter compensation for real-time control systems. In: Proceedings of the 22nd IEEE Real-Time Systems Symposium. London, England.

Palopoli, L., A. Bicchi and A. Sangiovanni Vincentelli (2002). Numerically efficient control of systems with communication constraints. In: Proceedings of the 41st IEEE Conference on Decision and Control. Las Vegas, USA.

Rehbinder, H. and M. Sanfridson (2004). Scheduling of a limited communication channel for optimal control. Automatica 30(3), 491-500.

Seto, D., J. P. Lehoczky, L. Sha and K. G. Shin (1996). On task schedulability in real-time control systems. In: Proceedings of the 17th IEEE Real-Time Systems Symposium. New York, USA.

Ye, Y. (1993). A fully polynomial-time approximation algorithm for computing a stationary point of the general linear complementarity problem. Mathematics of Operations Research 18(2), 334-345. 